\title{
Images in Clinical Medicine
}

\section{Tectal Plate Cavernous Malformation-A Rare Cavernoma in Brainstem}

Received: November 29, 2019 Accepted: December 31, 2019

doi: https://doi.org/10.3329/jemc.v10i1.45068

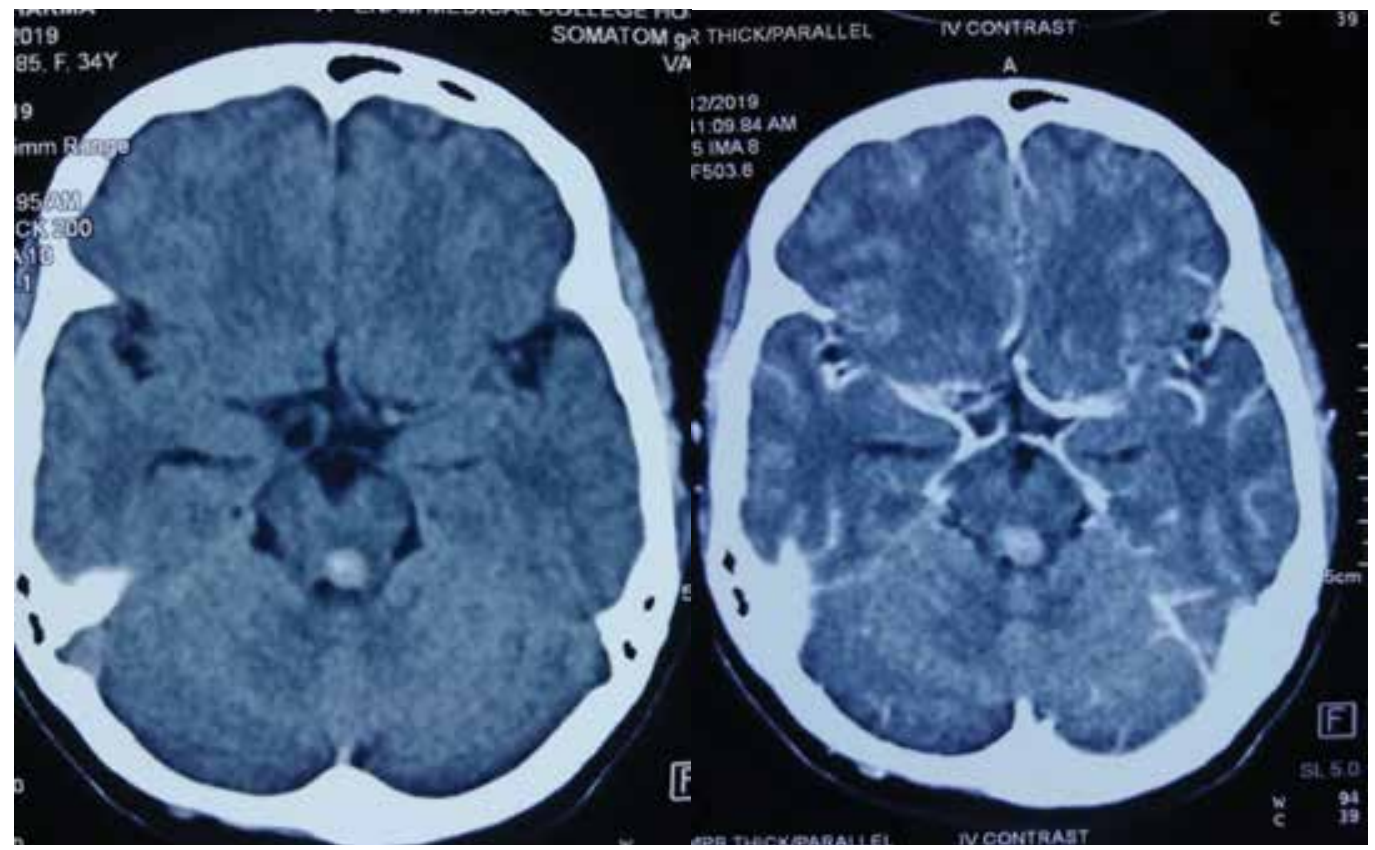

Fig 1. Pre- and post-contrast axial CT images showing enhancing dense opacity in tectum of midbrain

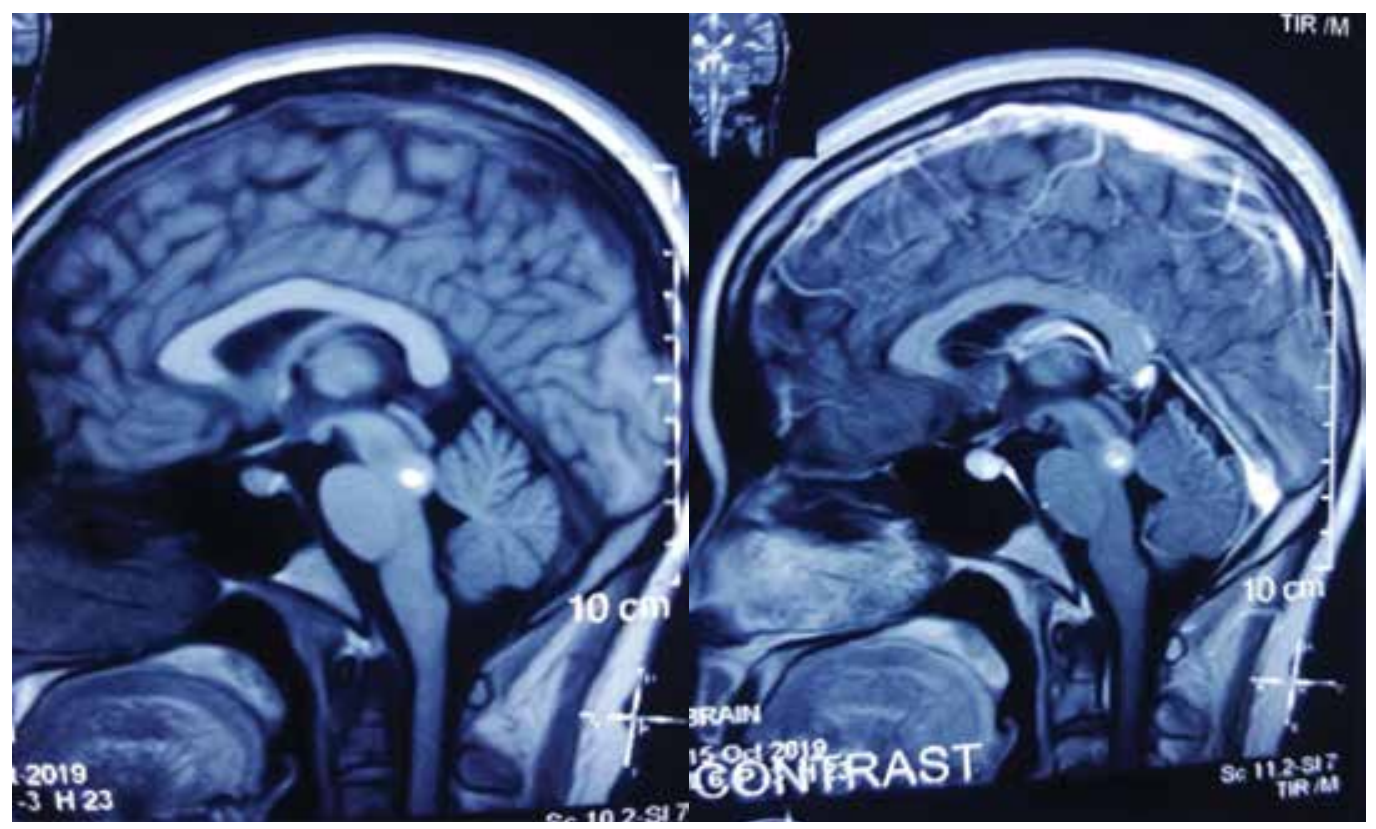

Fig 2. Pre- and post-contrast T1W sagittal images showing minimally enhancing hyperintense lesion in tectum of midbrain 


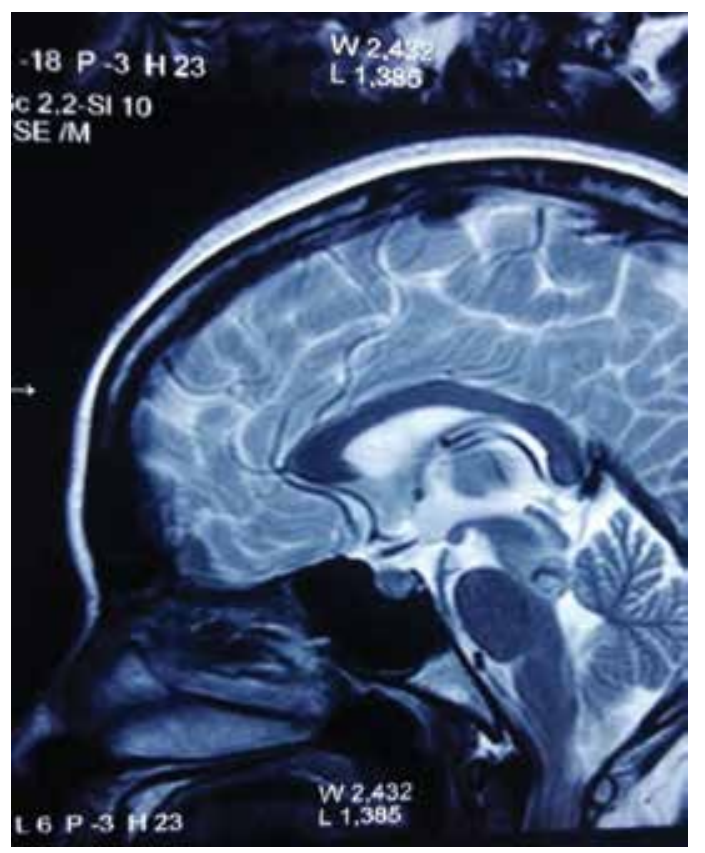

Fig 3. T2W sagittal image showing heterogeneous intense mass involving dorsal aspect of tectum of midbrain

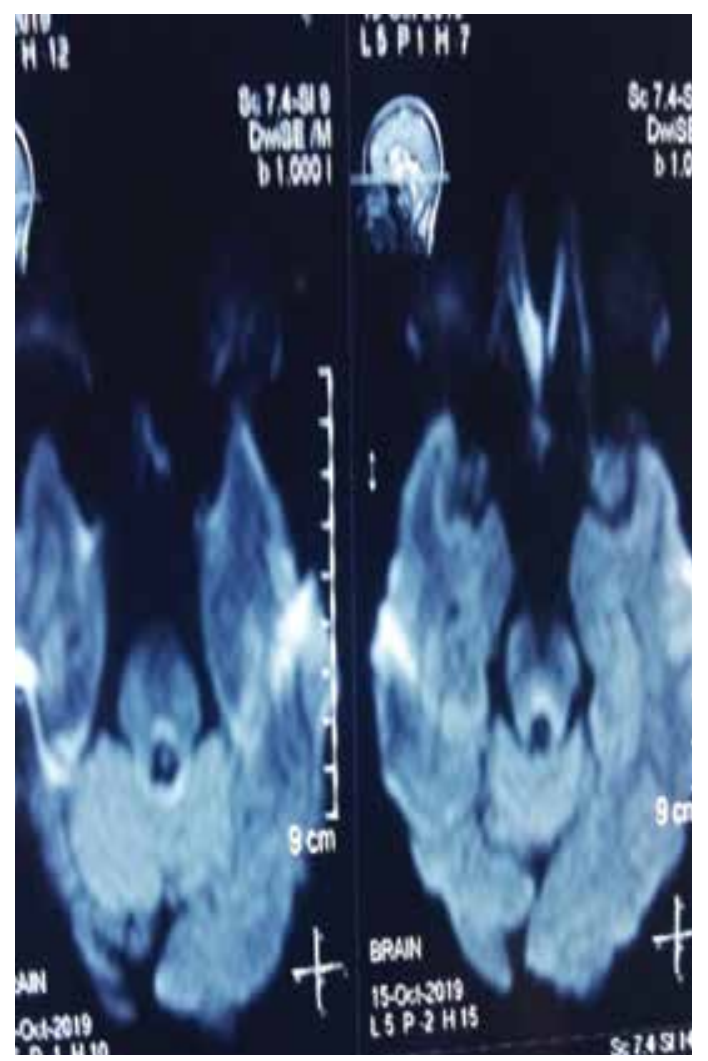

Fig 4. DW axial image showing hypointense lesion measuring about $9 \mathrm{~mm} \times 8 \mathrm{~mm}$ seen in tectum of midbrain

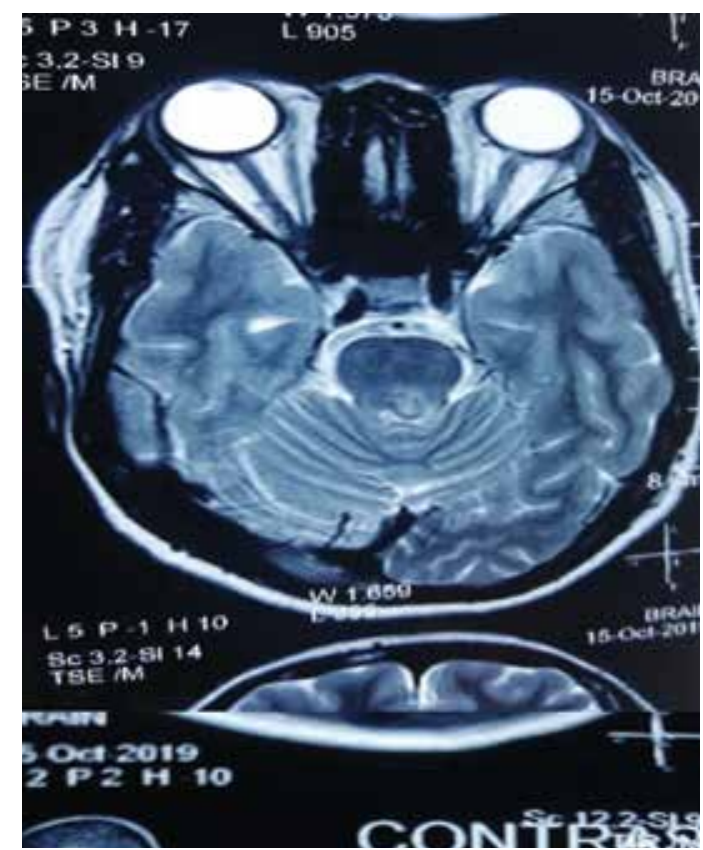

Fig 5. Post-contrast T2W axial image showing minimally enhancing mass in tectum of midbrain

A 34-year-old male patient attended the Department of Radiology \& Imaging in Enam Medical College Hospital for CT scan of brain. He complained of headache and vertigo for four years and occasional generalized weakness.

CT scan of brain revealed a well-defined regular outlined round densely calcified area with internal low signal foci in tectum of midbrain, measuring about $9 \mathrm{~mm} \times 8 \mathrm{~mm}$. After IV contrast, there was minimal diffuse enhancement. Pre-contrast max density was +69 HU. Post-contrast max density was +73 HU. There was only mild contour bulging at posterior margin of tectum. No compression over cerebral aqueduct of midbrain was seen. Besides this there was bilateral basal ganglia calcification which was physiological for the age of patient. So in CT scan provisional diagnosis was cavernous malformation at tectum of midbrain. MRI of brain with IV contrast was recommended for further evaluation.

MRI of brain revealed a small lesion $(9 \mathrm{~mm} \times 8$ $\mathrm{mm}$ ) in the dorsal region of midbrain involving the tectum. The lesion was hyperintese on T1W1 \& FLAIR image, hypointense on GRE and DW images, heterogeneous intense on T2W1. After I/V contrast minimal enhancement was noted. MRI findings were 
consistent with cavernous malformation in the tectum of midbrain.

Cavernous malformations are dilated blood vessels that are characterized by multiple distended "caverns" of blood-filled vasculature through which blood flows very slowly. Cavernous malformations are primarily located in the brain, but can also be found in the spinal cord, on the skin and more rarely in the retina. ${ }^{1}$

Cerebral cavernous malformations (CCMs) are usually located in the white matter (cortex) of the brain. CCMs do not have brain tissue within the malformation like other lesions such as arteriovenous malformations, and they usually are not encapsulated.

CCMs are present in up to $0.5 \%$ of the general population, and they account for a large proportion (8$15 \%$ ) of all brain and spinal vascular malformations. Locations in the tectal plate that represent a small percentage of brainstem CMs have rarely been reported in the literature. ${ }^{2}$

While adults are most often diagnosed, people of all ages may be affected by cavernous malformations, and approximately $25 \%$ of all diagnosed cavernous malformations are found in children. They are usually solitary although up to one-third of patients with sporadic lesions have more than one. Generally signs and symptoms of CCMs may include weakness, numbness, difficulty in speaking, difficulty in understanding others, unsteadiness, vision changes or severe headache. Seizures also can occur and neurological issues can progressively worsen over time with recurrent bleeding. ${ }^{1}$

Cavernous malformation in the tectum of midbrain is a heterogeneous group of malignant tumors of mesenchymal originating from the soft tissues rather than bone. They are classified on the basis of tissue seen on histology. Brainstem cavernous malformations have a high incidence of bleeding and rebleeding and carry a high rate of neurologic morbidity. ${ }^{3}$

MRI is now the standard of care for diagnosing cavernous malformations. Unless an individual has had a recent bleed, CCM lesions are not readily detected by CT scans or angiography. Molecular genetic testing for mutations in the KRIT1 (CCM1),
CCM2, and PDCD10 (CCM3) genes is available to confirm the diagnosis. ${ }^{3}$

Differential diagnosis is tectal glioma which falls under the grouping of childhood brainstem glioma which is a homogeneous expansion of tectal plate and isodense to grey matter. On MRI the tumor demonstrates expansion of the tectal plate by a solid nodule of tissue. On T1W1 lesion is iso to slightly hypointense to grey matter and on T2WI hyperintese to grey matter. In contrast, lesion usually shows no enhancement. ${ }^{2}$ Cavernous malformations are asymptomatic and can be treated conservatively. Symptomatic lesions when possible can be resected and complete resection is curative. ${ }^{4}$

\section{Samar C Sarkar}

Associate Professor

Department of Radiology \& Imaging

Enam Medical College \& Hospital, Savar, Dhaka

\section{Mashah Binte Amin}

Associate Professor

Department of Radiology \& Imaging

Enam Medical College \& Hospital, Savar, Dhaka

\section{References}

1. Doris L, Sachin B, Pablo F, Jun Z. Cavernous malformations: natural history, diagnosis and treatment. NR Neurol 2009; 5: 659-670.

2. Cristini A, Fischer C, Sindou M. Tectal plate cavernoma - a special entity of brainstem cavernomas: case report. Surg Neurol 2004; 61(5): 474-478.

3. Petersen TA, Morrison LA, Schrader RM, Hart BL. Familial versus sporadic cavernous malformations: differences in developmental venous anomaly association and lesion phenotype. AJNR Am J Neuroradiol 2010; 31(2): 377-382.

4. Giliberto G, Lanzino DJ, Diehn FE, Factor D, Flemming KD, Lanzino G. Brainstem cavernous malformations: anatomical, clinical, and surgical considerations. Neurosurg Focus 2010; 29(3): 9-19. 